Copyright (c) 2020 Fernanda Campos de Almeida Carrer, Bruno Matuck, Edson Hilan Gomes de Lucena, Fábio Carneiro Martins, Gilberto Alfredo Pucca Junior, Mariana Lopes Galante, Maria Fernanda de Montezuma Tricoli, Mary Caroline Skelton Macedo

(c) (1) Este trabalho está licenciado sob uma licença Creative Commons Attribution 4.0 International License. Fonte: https://preprints.scielo.org/index.php/scielo/preprint/view/837. Acesso em: 24 jun. 2020.

\title{
REFERÊNCIA
}

CARRER, Fernanda Campos de Almeida et al. Teleodontologia e SUS: uma importante ferramenta para a retomada da Atenção Primária à Saúde no contexto da pandemia de COVID-19. Scielo Preprints, 2020. DOI: https://doi.org/10.1590/SciELOPreprints.837. Disponível em:

https://preprints.scielo.org/index.php/scielo/preprint/view/837/1159. Acesso em: 25 jun. 2020. 

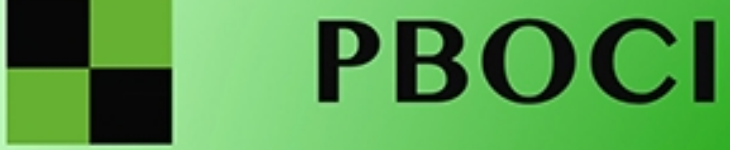

PESQUISA BRASILEIRA EM

ODONTOPEDIATRIA E CLINICA INTEGRADA

\section{Teleodontologia e SUS: uma importante ferramenta para a retomada da Atenção Primária à Saúde no contexto da pandemia de COVID-19}

\begin{tabular}{|c|c|}
\hline Journal: & $\begin{array}{l}\text { Pesquisa Brasileira em Odontopediatria e } \\
\text { Clínica Integrada }\end{array}$ \\
\hline Manuscript ID & PBOCI-2020-0140 \\
\hline Manuscript Type: & Short Communication \\
\hline $\begin{array}{l}\text { Keyword - Chosen from the keywords registered at Medical } \\
\text { Subject Headings of the U.S. National Library of Medicine } \\
(<\text { a href="https://meshb.nlm.nih.gov/search" } \\
\text { target="_blank" }>\text { https://meshb.nlm.nih.gov/search }</ a>) \text {.: }\end{array}$ & $\begin{array}{l}\text { Teledentistry, Telemedicine, Community } \\
\text { Dentistry, Public Policy, Coronavirus } \\
\text { Infections }\end{array}$ \\
\hline
\end{tabular}

\section{SCHOLARONE"}

Manuscripts 
Teleodontologia e SUS: uma importante ferramenta para a retomada da Atenção Primária à Saúde no contexto da pandemia de COVID-19

Fernanda Campos de almeida Carrer (Universidade de São Paulo- Faculdade de OdontologiaDepartamento de Odontologia Social. Av. Prof. Lineu Prestes, 2227- Cidade Universitária- São PauloSP/Brazil. ZIPCODE: 05508000. Phone/Fax: 5511 30917891. Email: fernandacsa@usp.br) Orcid: https://orcid.org/0000-0003-3745-2759

Bruno Fernandes Matuck (Universidade de São Paulo- Faculdade de Medicina- Departamento de Patologia. Av. Dr. Arnaldo, 455 - Cerqueira César, São Paulo - SP, ZIPCODE 01246-903. Phone/Fax: 5511 3061-7000. Email: bruno.matuck@ gmail.com) Orcid: https://orcid.org/0000-0002-2132-3402

Edson Hilan Gomes de Lucena (Universidade Federal da Paraíba - faculdade de Odontologia Departamento de Clínica e Odontologia Social. Campus I - Cidade Universitária ZIPCODE 58.051900 - João Pessoa-PB. Phone/fax: (83) 3216 7251. Email: ehglucena@gmail.com) Orcid: http://orcid.org/0000-0003-3431-115X.

Fábio Carneiro Martins (Universidade de São Paulo - Faculdade de Odontologia- Departamento de Odontologia Social. Av. Prof. Lineu Prestes, 2227- Cidade Universitária- São Paulo- SP/Brazil. ZIPCODE: 05508000. Phone/Fax: 5511 30917891. Email: fabio.carneiro.martins@usp.br) Orcid: https://orcid.org/0000-0002-7014-9439

Gilberto Alfredo Pucca Junior (Universidade de Brasília UnB - Faculdade de Ciências de Saúde Departamento de Odontologia. Asa Norte, Brasília - DF- ZIPCODE 70910-900 Phone/fax: (61) 31071940 / 1941 / 1942 Email: gilbertopucca@unb.br) Orcid: https://orcid.org/0000-0002-8781-9857

Mariana Lopes Galante (Universidade de São Paulo- Faculdade de Odontologia- Departamento de Odontologia Social. Av. Prof. Lineu Prestes, 2227- Cidade Universitária- São Paulo- SP/Brazil. ZIPCODE: 05508000. Phone/Fax: 5511 30917891. Email: marilopes8@gmail.com) Orcid: https://orcid.org/0000-0003-2072-7875

Maria Fernanda de Montezuma Tricoli (Secretaria do Estado de Saúde - São Paulo. Av. Dr. Enéas Carvalho de Aguiar, 188 - Cerqueira César, São Paulo - SP, ZIPCODE 05403-000 Email: mftricoli@saude.sp.gov.br Orcid: https://orcid.org/0000-0002-5252-5699

Mary Caroline Skelton Macedo (Universidade de São Paulo- Faculdade de OdontologiaDepartamento de Dentística. Av. Prof. Lineu Prestes, 2227- Cidade Universitária- São Paulo- SP/Brazil. ZIPCODE: 05508000. Phone/Fax: 5511 30917891. Email: mmacedo@uol.com.br) Orcid: https://orcid.org/0000-0002-4189-5088

Contribuição dos autores: todos os autores contribuíram da mesma forma com a elaboração do manuscrito. 
Teleodontologia e SUS: uma importante ferramenta para a retomada da Atenção Primária à Saúde no contexto da pandemia de COVID-19

\section{RESUMO}

Este "short communication" descreve as possibilidades de utilização da Teleodontologia para ampliação e qualificação do cuidado em saúde nas redes de atenção à saúde bucal. A OMS já recomendava aos seus países membros, mesmo antes da pandemia, o Telessaúde como estratégia para melhorar a qualidade dos serviços, especialmente nos sistemas universais, como é o caso do Sistema Único de Saúde. A Teleodontologia abre oportunidades para que a saúde bucal retome a oferta de diversos serviços, de forma remota, como: 1. rastreamento, busca ativa, monitoramento de usuários prioritários, de risco e com problemas sistêmicos, de suspeitas de COVID-19 e contactantes, através do Telemonitamento; 2. escuta inicial, atividades educativas individuais ou coletivas, através da Teleorientação; 3. discussão de casos clínicos para a definição da oportunidade/necessidade de procedimentos operatórios, matriciamento, compartilhamento, solução de dúvidas entre profissionais e entre estes e instituições de ensino e pesquisa, por Teleconsultorias, entre outros. Além de uma revisão sobre a Teleodontologia no contexto da pandemia, conceituamos os termos utilizados e possibilidades que oferecem aos profissionais do SUS, além de especificar os protocolos possíveis para registro dessas atividades, a fim de fornecer dados seguros para seu monitoramento e avaliação. Ademais, trazemos uma breve discussão com experiências promissoras, realizadas nos contextos pré e trans pandemia, que podem ser importantes estratégias para a retomada da saúde bucal no cenário pós pandemia.

Palavras-chave: Teleodontologia, Telemedicina, Odontologia Comunitária, Políticas Públicas, Infecções por Coronavirus 


\title{
Teledentistry and the Unified Health System: an important tool for the resumption of Primary Health Care in the context of the COVID-19 pandemic
}

\begin{abstract}
This "short communication" describes the possibilities of using Teleodontology to expand and qualify health care in oral health care networks. WHO already recommended to its member countries, including Brazil, even before the pandemic, the use of Telehealth as a strategy to improve the quality of its services, especially in the context of universal health systems, as the Unified Health System In the current context, Teleodontology opens opportunities for oral health to resume the offer of various services, remotely, such as: 1. tracking, active search, monitoring users of priority groups, at risk and with systemic problems, monitoring of suspected cases of COVID-19 and its contacts, via telemonitoration; 2. initial listening, individual and collective educational activities, among others, through teleorientation; 3. discussion of clinical cases to define the opportune moment to perform operative procedures among professionals of different levels of care, matrix support, sharing and soluting doubts between professionals or between professionals and teaching and research institutions, through teleconsulting, among others . In addition to a literature review on Teleodontology in the context of the pandemic, an important contribution of this "short communication" is the conceptualization of the terms used and possibilities they offer to SUS professionals, in addition to specifying the possible protocols for recording these activities, in order to provide service managers with secure data for monitoring and evaluating the work process. In addition, we bring a brief discussion with some promising experiences, carried out in the pre and transpandemic context, which can consolidate themselves as important strategies for the resumption of oral health in the post-pandemic scenario.
\end{abstract}

Keywords: Teledentistry. Telemedicine, Community Dentistry, Public Policy, Coronavirus infections. 


\section{INTRODUÇÃO}

Em 11 de março de 2020, a organização mundial de saúde (OMS) decretou estado de pandemia da COVID-19. Uma nova infecção pulmonar, que se iniciara na cidade de Wuhan (China) e causava sintomas de uma síndrome respiratória aguda grave ${ }^{1}$. $\mathrm{O}$ agente biológico causador de tal acometimento é um coronavírus, vírus conhecido por outras duas epidemias do século XXI: a SARS (2002) e a MERS (2011), que tiveram disseminação restrita ao sudeste asiático.

A COVID-19 se difere dessas outras epidemias em sua alta e rápida disseminação pelo mundo, tal eficácia está pautada em três pilares: 1 . Longo período de incubação 2 . Letalidade relativa 3. Alta transmissibilidade dos assintomáticos ${ }^{2}$.

A COVID-19 apresenta especial impacto na odontologia, à medida que estudos indicam sítios da cavidade oral como possíveis focos de entrada do coronavírus nas células do hospedeiro humano. Trabalhos realizados com outros coronavírus em animais, demonstram que receptores de angiotensina presentes nos ductos das glândulas salivares, podem ser o alvo primário de invasão celular do patógeno ${ }^{3}$.

O fato deste vírus estar presente dentro de células de tecidos bucais mitiga a chance de que bochechos pré-operatórios eliminem totalmente o coronavírus das secreções salivares e creviculares ${ }^{4}$. Isso faz com que o aerossol gerado durante os procedimentos odontológicos seja potencialmente contaminado.

Portanto, a atividade odontológica é uma das mais críticas, pois grande parte dos procedimentos operatórios gera aerossóis, soma-se a isto, a escassez de equipamentos de proteção individual, a falta de protocolos claros de biossegurança e a necessidade de preservar as equipes de saúde e reduzir os riscos de contaminação dos usuários, diante deste cenário, os sistemas de saúde suspenderam os procedimentos eletivos em odontologia, com manutenção apenas de urgências e emergências, em diversos países, conforme recomendado por diversas instituições e governos locais ${ }^{5-9}$.

Neste contexto, a Teleodontologia tem sido citada por artigos científicos ${ }^{10-13}$ e documentos governamentais, como uma alternativa para garantir cuidados em saúde à população. Além disso, no contexto do Sistema único de Saúde (SUS), as Equipes de Saúde Bucal (ESB) podem fazer uso deste tipo de ferramenta para realizar atividades que as integram à equipe multiprofissional no enfrentamento da pandemia, como o rastreamento, Teleorientação e Telemonitoramento dos usuários com suspeita de COVID-19 e seus contactantes. O objetivo deste "short communication" é descrever e analisar, com base nas melhores evidências científicas disponíveis, as possibilidades de atuação e estratégias de implementação da Teleodontologia, como ferramenta estratégica para a oferta do cuidado em saúde bucal no contexto da pandemia de COVID-19, observando as normas brasileiras, as diretrizes do Sistema Único de Saúde e as possibilidades de permanência deste cuidado mediado por tecnologia pós pandemia. 


\section{A TELEODONTOLOGIA: UMA BREVE REVISÃO DA LITERATURA}

A Teleodontologia tem se mostrado eficaz tanto no custo quanto na disseminação do acesso, sendo um meio de democratização e equidade, com vantagens relacionadas ao aumento da resolutividade, à redução do tempo de espera e dos custos de tratamento ${ }^{14-16}$. Uma revisão sistemática mostrou a sua relação custo-benefício com pacientes que vivem em áreas rurais, provando ser monetariamente eficaz, com a implementação da Teleodontologia nas práticas odontológicas. Este estudo também mostrou que a Teleodontologia pode ser eficiente na triagem de lesões orais, principalmente em programas escolares, em áreas rurais e com acesso limitado aos cuidados e instalações de cuidados prolongados ${ }^{17}$.

O uso das Tecnologias de Informação e Comunicação (TIC) ocorre como parte dos serviços de saúde pública odontológica em países da América Latina, como Brasil, Colômbia e Uruguai, para melhorar a educação continuada e pesquisa colaborativa entre instituições nacionais e estrangeiras ${ }^{16,18}$. No Brasil, através das TIC, foram trocadas informações entre universidades e profissionais da atenção básica, agregando valores para a relação ensinoserviço e sendo uma forma inovadora de atendimento e qualidade de serviço ${ }^{19}$. Essas tecnologias, que também são úteis para orientação de capacitações e atividades de educação continuada, neste momento de pandemia, têm sido utilizadas para pré-triagem, orientação de profissionais e pacientes, em países como Paraguai e Costa Rica ${ }^{20,21}$.

No entanto, há escassez de projetos de Teleodontologia nos países em desenvolvimento, que tem sido atribuída ao conservadorismo dos tomadores de decisão, à falta de recursos, infraestrutura e equipamentos de TIC ${ }^{22}$. Um outro problema é a prestação de serviços de atendimento odontológico ainda se basear em atendimento emergencial e curativo, com falta de protagonismo dos cuidados preventivos ${ }^{22}$. Com a crise da COVID-19, emergiu a necessidade de incorporação da Teleodontologia na rotina do atendimento odontológico, em especial no Sistema Único de Saúde ${ }^{23}$.

A pandemia introduziu preocupações extras aos profissionais e usuários dos serviços de saúde bucal, entre eles os pacientes com diagnóstico de câncer de cabeça e pescoço, considerando que: 1. alguns pacientes não estão preparados emocional ou psicologicamente para receber tratamentos que mudam a vida; 2 . os pacientes diagnosticados com câncer ou com suspeita de lesões malignas devem ser monitorados constantemente e; 3 . a falta de acompanhamento dentário pode levar a um aumento de casos não diagnosticados, causando danos futuros ao paciente devido ao atraso no diagnóstico ${ }^{24}$.

O uso das TIC para fornecer atendimento odontológico remotamente pode permitir que as ESB realizem triagem para atendimentos odontológicos de emergência e urgência, que evitem a ida desnecessária de usuários as unidades de saúde e forneçam serviços não essenciais, evitando contato próximo com os usuários. Por outro lado, aqueles que não oferecem este tipo de serviço devem continuar cumprindo sua obrigação profissional contínua de responder às perguntas e não abandonar os pacientes ${ }^{25}$.

Uma rapid review com recomendações para a reabertura de serviços odontológicos, concluiu que $94 \%$ das fontes fornecem informações sobre como agrupar pacientes, principalmente por telefone, para incluir a avaliação de risco do status potencial de COVID-19 (por exemplo, COVID-19 positivo, suspeito de COVID-19, necessidade / proteção especial assintomática), o que pode contribuir para o rastreamento e telemonitoramento de usuários doentes (sintomáticos ou assintomáticos) e seus contactantes. Alguns estudos também recomendam estratégias como a triagem de temperatura na recepção, consultas por telefone e 
vídeo, triagem por tela telefônica de todos os pacientes em busca de sinais ou sintomas de doença respiratória e avaliação sistemática do paciente no momento do check-in nas clínicas odontológicas $^{26}$.

\section{TERMOS FUNDAMENTAIS RELACIONADOS À TELEODONTOLOGIA: DEFINIÇÕES, POSSIBILIDADES DE ATUAÇÃO E REGISTRO NA ATENÇÃO PRIMÁRIA À SAÚDE}

Os dados são apresentados no Quadro 1.

\section{DISCUSSÃO}

As diversas especialidades que realizam procedimentos ambulatoriais envolvendo a região de cabeça e pescoço, tais como odontologia, otorrinolaringologia e oftalmologia, sofreram um "lockdown" com a instalação de uma nova pandemia, a COVID-19. Tal decisão foi pautada em dois pilares: 1) os atendimentos ambulatoriais relacionados a tais especialidades apresentam alto risco de contágio visto o íntimo contato do profissional de saúde com aerossóis potencialmente contaminados; 2) dificuldade na aquisição de equipamentos de proteção individual, que possibilitariam uma redução da exposição operador-paciente ${ }^{35}$.

Para mitigar o risco de contágio e ainda estimular medidas de distanciamento social, alternativas ao atendimento presencial rapidamente se mostram elegíveis. Em todo o mundo grande parte das profissões tem revisto suas práticas e na área da saúde, os atendimentos em telessaúde são citados pela literatura como alternativa assertiva e viável para garantir o acesso à rede de saúde pelos pacientes ${ }^{27}$.

No Brasil, medidas emergenciais foram tomadas, visando adequar o processo de trabalho dos serviços de saúde à nova realidade imposta pela pandemia. A maioria dos conselhos adotaram uma abordagem cautelosa a fim de garantir o cuidado em saúde mediado pelas tecnologias de informação e comunicação, mas restringindo as atividades possíveis. No caso do CFM, mesmo que restringido ao momento atual, a resolução permite a realização de teleconsultas, diagnóstico, telemonitoramento, teleinterconsulta e teleorientação ${ }^{36}$.

No que diz respeito ao CFO, tardiamente, foi publicada normativa que tornou expressamente vedado o exercício da odontologia à distância, mediado por tecnologias, para fins de consulta, diagnóstico, prescrição e elaboração de plano de tratamento odontológico. Entretanto, foram admitidas atividades de telemonitoramento e teleorientação, desde que não sejam realizadas por centrais de atendimento ou qualquer outro meio que centralize o recebimento de demandas e as distribua automaticamente ${ }^{30}$.

Além disso, através da normativa, foi vedada às operadoras de planos de saúde odontológicos e demais pessoas jurídicas, a veiculação de publicidade e propaganda utilizando o termo "Teleodontologia", termo internacional que engloba outras expressões aqui discutidas na odontologia e que serão estratégicas para a sua retomada no "novo mundo", principalmente no contexto da Atenção Primária à Saúde (APS). Essa segregação do termo "Teleodontologia" tira o Brasil de um cenário mundial, no qual se discute o cuidado em saúde bucal mediado por tecnologia em diversos níveis de atenção à saúde e cabe à academia e aos grupos que militam e estudam telessaúde e teleodontologia no Brasil resistir e reafirmar a Teleodontologia como área do conhecimento e ferramenta de cuidado em saúde. 
Ainda que a resolução do CFO necessite ser revista e precise contemplar as necessidades do mundo trans e pós-pandemia, mesmo com todas as limitações, esta resolução já permite uma retomada da odontologia com as ferramentas do telemonitoramento. Se monitorar é vigiar, a equipe de saúde bucal pode realizar "exames clínico, mediado por tecnologia, com finalidade epidemiológica", então é urgente que se desenvolva ferramentas capazes de fazer triagem de risco, busca ativa do paciente com suspeita de câncer de boca, cuidado remoto dos acamados e dos pacientes com necessidades especiais, no sentido de "orientar" familiares e usuários com uso de TIC. Ainda, respeitando os limites impostos pela resolução do CFO, é possível que as equipes realizem "escuta inicial", mediada por tecnologia, a fim de orientar o usuário, realizar pré-triagem, organizar agenda e o fluxo nas unidades para evitar acúmulo dos pacientes em salas de espera ${ }^{30,35}$. Vale ressaltar ainda que os gestores vão enfrentar problemas para registrar parte desses procedimentos nos sistemas de informação, já que muitos deles são classificados como "consultas".

Desde o início da pandemia, observamos constantemente cálculos em todos os veículos de comunicação sobre a necessidade de leitos de UTI, o que colabora com o entendimento da população sobre a gravidade do problema. Entretanto, apesar de o Protocolo do Ministério da Saúde informar que $80 \%$ dos casos serão atendidos na APS ${ }^{37}$, pouco tem sido discutido sobre o impacto na APS, que é o nível de atenção à saúde em que os profissionais atuam próximo aos territórios, às famílias que ali residem e aos determinantes sociais ${ }^{38}$. É na APS que podemos conscientizar as pessoas sobre sua responsabilidade social no momento que estamos vivendo, que podemos monitorar os casos suspeitos e seus contactantes, mesmo com a disponibilidade de poucos testes para diagnóstico, e organizar atividades de promoção de saúde ${ }^{39}$. Ademais, a Nota Técnica No 9/2020-CGSB/DESF/SAPS/MS afirma que a ESB “os profissionais de saúde bucal, como corresponsáveis pelo cuidado da população e integrantes das equipes multiprofissionais, deverão compor a equipe que realizará as ações do FAST-TRACK COVID$19^{*}{ }^{35}$, entretanto, os gestores municipais têm enfrentado dificuldade à medida que não há como registrar a produção desses profissionais nos sistemas de informação, tendo em vista que não houve ajustes, por parte do Ministério da Saúde, nesses sistemas.

Diante da paralisação dos atendimentos eletivos, cabe uma reflexão: "Qual o significado do cuidado odontológico na saúde do usuário?” A Odontologia poderá deixar de atender os pacientes com doenças crônicas (cardíacos, diabéticos, tabagistas e etilistas)? Como fazer busca ativa de câncer de boca no contexto do isolamento social? Como fazer atividades do Programa de Saúde na Escola (PSE) sem aulas presenciais?

Não há dúvidas que a Teleodontologia pode ser uma ferramenta potente para retomada segura do cuidado em saúde bucal no Sistema Único de Saúde, já que no contexto da saúde pública temos que planejar as ações com base nos princípios da universalidade, equidade e integralidade.

No Rio Grande do Sul, por exemplo, há um serviço muito eficiente disponível aos dentistas do Sistema Único de Saúde, chamado "EstomatoNet", que recebe demanda de dentistas e médicos da atenção primária, com envio de perguntas, dados clínicos e até fotos, para auxiliar no diagnóstico e na condução dos casos ${ }^{40}$. Este mesmo grupo chegou a usar a plataforma Whatsapp para trocar informação entre os profissionais da atenção básica e os teleconsultores do programa telessaúde redes do Rio Grande do Sul ${ }^{41}$.

Ainda sobre o programa Telessaúde Brasil Redes, é importante reforçar que houve grande investimento nos últimos anos para disseminação de Núcleos pelo território nacional e atualmente o Sistema Único de Saúde conta com uma malha qualificada e capilarizada que pode ser utilizada para impulsionar a teleodontologia no Sistema Único de Saúde, em todos os níveis 
de atenção, com ênfase para a atenção primária à saúde, que é o nível capaz de realizar medidas de prevenção, promoção e monitoramento dos grupos de risco.

No Núcleo Telessaúde Redes Unifesp, as perguntas sobre saúde bucal representam $32,9 \%$ de todas teleconsultorias, portanto a odontologia não é desprezível e merece ser observada pelo Conselho que regula o exercício da Odontologia, pelos tomadores de decisão e formuladores de políticas públicas de saúde, no contexto da teleodontologia. No que diz respeito à "Proposta de apoio emergencial à coordenação da saúde bucal do Estado de São Paulo diante da pandemia do COVID19", observa-se que os trabalhadores do SUS estão dispostos e motivados a discutir e participar de atividades de educação permanente que respondam às demandas de qualificação das ESB em face da pandemia da COVID-19, à medida que a gestão estadual mostra compromisso com seus trabalhadores e grande articulação com coordenadores municipais de saúde bucal e demonstram preocupação com a séria situação que a odontologia enfrenta no SUS. Em relação às dúvidas dos trabalhadores e gestores do SUS, observa-se que a maioria se concentra na biossegurança, o que é natural, à medida que a Odontologia é umas das profissões que mais sofre com este tema na sua prática profissional em face das rotas de transmissão do novo coronavírus.

Há fortes evidências no manejo de doenças crônicas em outras áreas da saúde, em campos distintos como a psiquiatria e a dermatologia. Exames com alta sensibilidade e especificidade demonstram a eficácia do teleatendimento em áreas clínicas e que realizam procedimentos ambulatoriais ${ }^{42}$. No âmbito da prática de telecardiológica, há relação eficaz na resolutividade dos casos atendidos, de alta e baixa complexidade, com melhoria na atenção da saúde de regiões onde tal atenção foi instalada. Estudos mostram que a telemedicina apresenta aumento da adesão do tratamento por parte dos pacientes, além de importante redução nos custos da atenção terciária. A implementação de teleatendimentos no âmbito das especialidades pode representar uma economia de até 3546 euros para hospitais e centros de referência ${ }^{43}$. A relação de custo-morbidade que as especialidades médicas vivenciam apresentam um limiar de tolerância menor do que os da realidade odontológica, mesmo assim, a prática da Teleodontologia ainda não se mostra tão sedimentado quanto a Telemedicina.

\section{CONSIDERAÇÕES FINAIS}

Em um contexto global complexo, caracterizado pela maior crise sanitária contemporânea imposta pela pandemia causada pelo novo coronavírus, somada, no Brasil, com uma grave crise política, o atraso no diagnóstico e acúmulo de necessidades são problemas que podem causar um impacto financeiro e social importante para os serviços de saúde e seus usuários, e o uso da Teleodontologia tem um grande potencial de enfrentamento, pois permite a redução de barreiras geográficas em um país com dimensões territoriais extensas, bem como o fortalecimento da APS. O Sistema Único de Saúde tem compromisso constitucional com a universalidade do acesso, observando a equidade das ações e a integralidade do cuidado, portanto, cabe aos trabalhadores e gestores deste sistema dar assistência, que no contexto da pandemia só pode ser viável com uso das TIC. O telemonitoramento e teleorientação já permitem uma retomada parcial do cuidado em saúde bucal da APS no SUS, mas é preciso que se revise a portaria com inclusão de procedimentos como consulta e prescrição, para aumentar as possibilidades da atuação dos profissionais do SUS. Vale ressaltar que as TIC podem ser grandes aliadas para aumentar acesso, neste mundo cada vez mais digital, lutar contra esta realidade pode significar um atraso que vai ser cobrado pela história. 


\section{AGRADECIMENTOS}

Laboratório UFPB, RPN, UNIFESP, SES/SP, FOUSP, TELESSAÚDE BRASIL REDES UNIFESP

\section{REFERÊNCIAS}

1- Zhu N, Zhang D, Wang W, et al. A Novel Coronavirus from Patients with Pneumonia in China, 2019. N Engl J Med. 2020;382(8):727-733. doi:10.1056/NEJMoa2001017

2- Lauer SA, Grantz KH, Bi Q, et al. The Incubation Period of Coronavirus Disease 2019 (COVID-19) From Publicly Reported Confirmed Cases: Estimation and Application. Ann Intern Med. 2020;172(9):577-582. doi:10.7326/M20-0504

3 - Liu L, Wei Q, Alvarez X, et al. Epithelial cells lining salivary gland ducts are early target cells of severe acute respiratory syndrome coronavirus infection in the upper respiratory tracts of rhesus macaques. J Virol. 2011;85(8):4025-4030. doi:10.1128/JVI.02292-10

5. Ministério da Saúde (BR). Secretaria de Atenção Primária à Saúde (SAPS). Atendimento odontológico no SUS. Brasília, DF; 2020. [Acessado em 08 de junho de 2020] Available from: http://www.crosp.org.br/uploads/arquivo/ab69d79b87d04780af08a70d8cee9d70.pdf

6. Argentina, Ministério de la Salud Covid-19 ATENCIÓN ODONTOLÓGICA PROGRAMADA INICIAL. [Acessado em 08 de junho de 2020]. Disponível em http://www.msal.gob.ar/images/stories/bes/graficos/0000001937cnt-covid-19recomendaciones-atencion-odontologica-programada.pdf.5.

7. Chile, MINSAL ORIENTACIONES PARA ATENCIÓN ODONTOLÓGICA EN FASE IV COVID-19. [Acessado em 08 de junho de 2020]. Disponível em: https://diprece.minsal.cl/wp-content/uploads/2020/03/ORIENTACIONES-ATENCIONODONTOLOGICAS-COVID-19-.pdf.6.

8. American Dental Association - ADA. ADA recommending dentists postpone elective procedures. American Dental Assocaition. 2020. [Acessado em 08 de junho de 2020] Disponível em: https://www.ada.org/en/publications/ada-news/2020-archive/march/adarecommending-dentists-postpone-elective-procedures [ Links]

9. PEREIRA, Luciano José et al. Biological and social aspects of Coronavirus Disease 2019 (COVID-19) related to oral health. Braz. oral res. [online]. 2020, vol.34 [cited 2020-06-08], e041. Available from: <http://www.scielo.br/scielo.php?script=sci_arttext\&pid=S180683242020000100600\&lng=en\&nrm=iso>. Epub May 08, 2020. ISSN 1807-3107. https://doi.org/10.1590/1807-3107bor-2020.vol34.0041.

10. Kochhar AS, Bhasin R, Kochhar GK, Dadlani H. Provision of continuous dental care for oral oncology patients during \& after COVID-19 pandemic. Oral Oncol. 2020;106:104785. doi:10.1016/j.oraloncology.2020.104785

11. Day AT, Sher DJ, Lee RC, et al. Head and neck oncology during the COVID-19 pandemic: Reconsidering traditional treatment paradigms in light of new surgical and other multilevel risks. Oral Oncol. 2020;105:104684. doi:10.1016/j.oraloncology.2020.104684

12. Győrffy Z, Békási S, Szathmári-Mészáros N, Németh O. A telemedicina lehetőségei a COVID-19-pandémia kapcsán a nemzetközi és a magyarországi tapasztalatok és ajánlások tükrében: (A COVID-19-pandémia orvosszakmai kérdései) [Possibilities of telemedicine 
regarding the COVID-19 pandemic in light of the international and Hungarian experiences and recommendations]. Orv Hetil. 2020;161(24):983-992. doi:10.1556/650.2020.31873

13. Giudice A, Barone S, Muraca D, et al. Can Teledentistry Improve the Monitoring of Patients during the Covid-19 Dissemination? A Descriptive Pilot Study. Int J Environ Res Public Health. 2020;17(10):E3399. Published 2020 May 13. doi:10.3390/ijerph17103399

14. Gullbrandsson L, Eklund B, Kildal M, et al. Telemedicine-A Complement to Traditional Referrals in Oral Medicine Lena. Telemed J E Heal 2012; 18: 549-553.

15. Estai M, Bunt SM, Kanagasingam Y, et al. A resource reallocation model for school dental screening : taking advantage of teledentistry in low-risk areas. Int Dent J 2018; 1-7.

16. Bo C, Peralta S, Lu A. How Has Teledentistry Been Applied in Public Dental Health Services? An Integrative Review. 2019; 00: 1-10.

17. Alabdullah JH, Daniel SJ. A systematic review on the validitidy of teledentistry. 2018; 24 : $1-10$.

18. Jordi CL, Figueiredo MÇ, Barone D, et al. STUDY AND ANALYSIS OF INFORMATION TECHNOLOGY IN DENTISTRY IN LATIN AMERICAN COUNTRIES. 2016; 29: 14-22.

19. Tonini K, Nascimento RM, Rios MZ. Information and communication technologies for professional training in Dentistry : a Telehealth / ES proposal. Rev da ABENO 2018; 18: $127-$ 136.

20. Paraguay Ministerio de Salud Publica y Bienestar Social. ATENCIÓN ODONTOLÓGICA DURANTE LA PANDEMIA DE SARS-CoV-2 en la REPUBLICA DEL PARAGUAY.

21. Colegio Cirujanos Dentistas Costa Rica. Protocolo Teleconsulta Dental.

22. Estai M, Kanagasingam Y, Tennant M, et al. A systematic review of the research evidence for the benefits of teledentistry. J Telemed Telecare 2017; 24: 147-156.

23. Villa A, Sankar V, Shiboski C. Tele ( oral ) medicine: A new approach during the COVID-19 crisis. Oral Dis 2020; $1-2$.

24. Rogers AKSN. The After Diagnosis Head and Neck cancer - specific Patient Concerns Inventory ( HaNC - AD ) as a pre - treatment preparation aid during the COVID - 19 pandemic. Eur Arch Oto-Rhino-Laryngology. Epub ahead of print 2020. DOI: 10.1007/s00405-02005995-9.

25. Royal College of Dental Surgeons of Ontario. COVID-19: Guidance for the Use of Teledentistry, https://www.rcdso.org/en-ca/rcdso-members/2019-novel-coronavirus/covid-19--emergency-screening-of-dental-patients-using-teledentistry (2020).

26. CoDer Working Group. Recommendations for the re-opening of dental services : a rapid review of international sources. Cochrane Database Syst Rev.

27. Global diffusion of eHealth: making universal health coverage achievable. Report of the third global survey on eHealth. Geneva: World Health Organization; 2016. Licence: CC BYNC-SA $3.0 \quad$ IGO. Disponível em: https://apps.who.int/iris/bitstream/handle/10665/252529/9789241511780-eng.pdf?sequence=1

28. Khan SA, Omar H. Teledentistry in practice: literature review. Telemed J E Health. 2013;19(7):565-567. doi:10.1089/tmj.2012.0200 
29. LOPES, Marcelo Antônio Cartaxo Queiroga et al. Diretriz da Sociedade Brasileira de Cardiologia sobre Telemedicina na Cardiologia - 2019. Arq. Bras. Cardiol. [online]. 2019, vol.113, n.5 [cited 2020-06-08], pp.1006-1056. Available from: $<$ http://www.scielo.br/scielo.php?script=sci_arttext\&pid=S0066-

782X2019001101006\&lng=en\&nrm=iso>. Epub Dec 02, 2019. ISSN 1678-4170. http://dx.doi.org/10.5935/abc.20190205.

30. Conselho Federal de Odontologia. RESOLUÇÃO CFO-226. Dispõe sobre o exercício da Odontologia a distância, mediado por tecnologias, e dá outras providências. 04 de junho de 2020. [Acessado em 05 de junho de 2020] Disponível em: http://sistemas.cfo.org.br/visualizar/atos/RESOLU\%C3\%87\%C3\%83O/SEC/2020/226

31. Ferrer J, Nicuesa M, Esteban D, Ayala R (Editorial Conceitos). Monitoramento. Site: Conceitos.com. Publicado: 02/01/2017 [Acessado em 04 de junho de 2020]. Disponível em https://conceitos.com/monitoramento/

32. CONSULTA. In.: Dicio, Dicionário Online de Português. Porto: 7Graus, 2020. [Acessado em 04 de junho de 2020]. Disponível em https://www.dicio.com.br/consulta/

33. Deldar K, Bahaadinbeigy K, Tara SM. Teleconsultation and Clinical Decision Making: a Systematic Review. Acta Inform Med. 2016;24(4):286-292. doi:10.5455/aim.2016.24.286-292

34. BRASIL. Ministério da Saúde. PORTARIA Nº 2.546, DE 27 DE OUTUBRO DE 2011. Redefine e amplia o Programa Telessaúde Brasil, que passa a ser denominado Programa Nacional Telessaúde Brasil Redes (Telessaúde Brasil Redes). [Acessado em 05 de junho de 2020]. $\quad$ Disponível https://bvsms.saude.gov.br/bvs/saudelegis/gm/2011/prt2546_27_10_2011.html

35. BRASIL. Ministério da Saúde. Secretaria de Atenção Primária à Saúde (SAPS). NOTA TÉCNICA No 9/2020-CGSB/DESF/SAPS/MS. COVID-19 E ATENDIMENTO ODONTOLÓGICO NO SUS. Março de 2020. Disponível em: http://website.cfo.org.br/wpcontent/uploads/2020/03/COVID-19_ATENDIMENTO-ODONTOLOGICO-NO-SUS.pdf

36. Conselho Federal de Medicina. OFÍCIO CFMNº1756/2020-COJUR. Informa sua decisão de reconhecer a possibilidade e a eticidade de uso da telemedicina no País. 19de março de 2020. [Acessado em 05 de junho de 2020] Disponível em: http://portal.cfm.org.br/images/PDF/2020_oficio_telemedicina.pdf

37. BRASIL. Ministério da Saúde. Secretaria de Atenção Primária à Saúde (SAPS). PROTOCOLO DE MANEJO CLÍNICO DO CORONAVÍRUS ( COVID-19) NA ATENÇÃO PRIMÁRIA. 2020.2 Disponíveis em: https://portalarquivos.saude.gov.br/images/pdf/2020/April/08/20200408-ProtocoloManejover07.pdf

38. Vitória AM, Campos GWS. Só com APS forte o sistema pode ser capaz de achatar a curva de crescimento da pandemia e garantir suficiência de leitos UTI. 13 de Abril de 2020. [Acessado em 03 de junho de 2020) disponível em: http://www.cosemssp.org.br/wpcontent/uploads/2020/04/So-APS-forte-para-ter-leitos-UTI-.pdf

39. STARFIELD, B. (1998). Primary care: balancing health needs, services, and technology. New York, Oxford University Press.

40. Carrard, V. C., Roxo Gonçalves, M., Rodriguez Strey, J., Pilz, C., Martins, M. A. T., Martins, M. D., ... \& Harzheim, E. (2018). Telediagnosis of oral lesions in primary care: The EstomatoNet Program. Oral diseases, 24(6), 1012-1019 
41. Carrard, V. C., Martins, M. A. T., Molina-Bastos, C. G., \& Gonçalves, M. R. (2017). WhatsApp: a telemedicine platform for facilitating remote oral medicine consultation and improving clinical examinations-some considerations. Oral surgery, oral medicine, oral pathology and oral radiology, 123(3), 408.

42. Hersh W,Helfand M, Wallace J, et al. A systematic review of the efficacy of telemedicine for making diagnostic and management decisions. J Telemed Telecare. 2002;8(4):197-209.

43. Comín-Colet J, Enjuanes C, Verdú-Rotellar JM, et al. Impact on clinical events and healthcare costs of adding telemedicine to multidisciplinary disease management programmes for heart failure: Results of a randomized controlled trial. J Telemed Telecare. 2016;22(5):282295. doi:10.1177/1357633X15600583 
Quadro 1 - Termos fundamentais em Teleodontologia, possibilidades de atuação e registro na Atenção Primária à Saúde (APS)

\begin{tabular}{|c|c|c|c|}
\hline Termo & Definição & Possibilidades na APS & Como registrar os procedimentos \\
\hline Telessaúde & $\begin{array}{l}\text { Provisão de serviços associados aos cuidados } \\
\text { com a saúde, nos casos em que a distância é } \\
\text { um fator crítico. Tais serviços podem ser } \\
\text { providos por meio do uso de tecnologias de } \\
\text { comunicação, com os objetivos de educação } \\
\text { continuada ou troca de informações } \\
\text { necessárias para diagnosticar, tratar, } \\
\text { prognosticar e prevenir doenças }{ }^{27} \text {. }\end{array}$ & $\begin{array}{l}\text { Cursos de ensino a distância (EAD), troca de conhecimentos, } \\
\text { orientação profissional e estudos de doenças epidemiológicas } \\
\text { locais a distância são exemplos de soluçôes viabilizadas pela } \\
\text { telessaúde. }\end{array}$ & $\begin{array}{l}\text { Compila todos ou outros campos, que serão descritos } \\
\text { individualmente }\end{array}$ \\
\hline Teleodontologia & $\begin{array}{lll}\text { Provisão remota } & \text { de } & \text { atendimento } \\
\text { odontológico } & & \\
& \end{array}$ & $\begin{array}{l}\text { Aconselhamento ou tratamento por meio de tecnologia da } \\
\text { informação, em vez de contato pessoal direto com qualquer } \\
\text { paciente envolvido }{ }^{28} \text {. }\end{array}$ & $\begin{array}{l}\text { Compila todos ou outros campos, que serão descritos } \\
\text { individualmente }\end{array}$ \\
\hline Telemedicina & Provisão remota de atendimento médico ${ }^{29}$. & $\begin{array}{l}\text { Aconselhamento ou tratamento por meio de tecnologia da } \\
\text { informação, em vez de contato pessoal direto com qualquer } \\
\text { paciente envolvido. Refere-se especificamente a serviços } \\
\text { clínicos à distância } 29 \text {. Inclui atendimento pré-clínico, de } \\
\text { suporte assistencial, de consulta, monitoramento e } \\
\text { diagnóstico, por meio de tecnologia da informação e } \\
\text { comunicação, no âmbito do SUS, bem como na saúde } \\
\text { suplementar e privada. Compreende o diagnóstico e realização } \\
\text { de conduta via emissão de receitas e atestados médicos à } \\
\text { distância será válida em meio eletrônico }\end{array}$ & Não se aplica para Odontologia. \\
\hline Teleorientação & $\begin{array}{l}\text { Identificação, através de questionário pré- } \\
\text { clínico, o melhor momento para a realização } \\
\text { do atendimento presencial }{ }^{30} \text {. }\end{array}$ & $\begin{array}{l}\text { Acolhimento, triagem, escuta inicial e orientação, para } \\
\text { soluções de dúvidas, esclarecimentos e realização de } \\
\text { atividades educativas individuais e coletivas. Também pode } \\
\text { ser utilizada para definir o momento mais oportuno para } \\
\text { realização de procedimentos operatórios. }\end{array}$ & $\begin{array}{l}\text { Na ficha de atendimentos odontológicos do e-SUS: } \\
\text { Local de atendimento } \\
\text { - Outros } \\
\text { Tipo atendimento } \\
\text { - Escuta inicial/orientação } \\
\text { Procedimentos (quantidade realizada) } \\
\text { - Orientação de higiene bucal } \\
\text { Conduta/Desfecho } \\
\text { - Retorno para consulta agendada; ou Alta do } \\
\quad \text { episódio. }\end{array}$ \\
\hline Telemonitoramento & $\begin{array}{l}\text { Monitoramento a distância de parâmetros de } \\
\text { saúde e/ou doença de pacientes, através das } \\
\text { TIC, podendo incluir a coleta de dados } \\
\text { clínicos, a transmissão, o processamento e o } \\
\text { manejo por um profissional de saúde } \\
\text { utilizando sistema eletrônico }{ }^{31} \text {. }\end{array}$ & $\begin{array}{l}\text { Supervisionar o estado de saúde dos usuários dos territórios de } \\
\text { interesse, ferramenta facilitadora para estratégias de } \\
\text { rastreamento de famílias com usuários que tenham fatores de } \\
\text { risco comum para doenças crônicas não transmissíveis } \\
\text { (tabagistas, etilistas etc.). No contexto da pandemia de } \\
\text { COVID-19, pode ser utilizada para controle do isolamento, } \\
\text { quarentena e monitoramento dos casos suspeitos e seus } \\
\text { contactantes; transmitir dados de um lugar a outro; realizar }\end{array}$ & $\begin{array}{l}\text { Na ficha de atendimentos odontológicos do e-SUS: } \\
\text { Local de atendimento } \\
\bullet \quad \text { Outros } \\
\text { Tipo atendimento } \\
\bullet \quad \text { Escuta inicial/orientação } \\
\text { Vigilância em saúde bucal } \\
\qquad \quad \text { Não identificado procedimento específico } \\
\quad \text { (importante que o MS faça adequação*) }\end{array}$ \\
\hline
\end{tabular}




\begin{tabular}{|c|c|c|c|}
\hline & & $\begin{array}{l}\text { intervenções à distância (na terminologia médica se fala do } \\
\text { monitoramento respiratório, cardíaco, neurológico, em relação } \\
\text { ao parto, entre outros) }{ }^{31} \text {. }\end{array}$ & $\begin{array}{l}\text { Procedimentos (quantidade realizada) - Outros } \\
\text { * Necessidade de adequação: acompanhamento e } \\
\text { monitoramento de usuários com fatores risco para } \\
\text { DCNT, Busca Ativa e Exame com finalidade } \\
\text { epidemiológica. } \\
\text { Conduta/Desfecho } \\
\text { - Retorno para consulta agendada; ou } \\
\text { - Alta do episódio. } \\
\text { *vale ressaltar que essas adequações são } \\
\text { administrativas, nos sistemas de informação, e não } \\
\text { geram impacto orçamentário. }\end{array}$ \\
\hline Teleconsulta & $\begin{array}{l}\text { Consulta significa atendimento; ação de } \\
\text { atender, diagnosticar ou receitar determinado } \\
\text { medicamento ou tratamento: consulta médica } \\
\text { 32. Teleconsulta é a consulta síncrona ou } \\
\text { assíncrona usando a tecnologia da } \\
\text { informação e comunicação para omitir a } \\
\text { distância geográfica e funcional }{ }^{33} \text {. }\end{array}$ & $\begin{array}{l}\text { Diagnóstico ou tratamento entre dois ou mais profissionais de } \\
\text { saúde separados geograficamente ou entre profissionais de } \\
\text { saúde e pacientes. }\end{array}$ & $\begin{array}{l}\text { Não se aplica (ainda não foi regularizada pelo CFO) } \\
\text { *Necessidade de adequação: flexibilização da } \\
\text { resolução para o SUS: a maior parte dos atendimentos } \\
\text { que não são procedimentos operatórios, são } \\
\text { registrados no e-SUS como algum tipo de consulta. }\end{array}$ \\
\hline Teleconsultoria & $\begin{array}{l}\text { Consulta realizada entre trabalhadores, } \\
\text { profissionais e gestores da área de saúde, } \\
\text { por meio de instrumentos de } \\
\text { telecomunicação bidirecional }{ }^{34} \text {. }\end{array}$ & $\begin{array}{l}\text { Esclarecimento de dúvidas sobre procedimentos clínicos, } \\
\text { ações de saúde e questões relativas ao processo de trabalho }{ }^{34} \text {. } \\
\text { As Teleconsultorias, os Telediagnósticos e a Segunda Opinião } \\
\text { Formativa são respondidas pelos Núcleos Técnico-Científicos } \\
\text { do Telessaúde Brasil Redes, com base na melhor e na mais } \\
\text { atualizada evidência clínica e científica disponível, adequada } \\
\text { ao contexto de origem da solicitação, realizada por } \\
\text { trabalhadores das Redes de Atenção à Sauude no SUS, em prazo } \\
\text { máximo de } 72 \text { horas. } \\
\text { Em municípios que não há a oferta destes serviços, a } \\
\text { ferramenta pode ser utilizada para que os profissionais dos } \\
\text { diversos níveis de atenção definam momento oportuno para } \\
\text { realização de encaminhamentos, priorizando atendimentos } \\
\text { urgentes, que não se limitam à dor, e evitando } \\
\text { encaminhamentos desnecessários. } \\
\text { O CFO veda uso da odontologia a distância, mediada por } \\
\text { tecnologias, para fins de consulta, diagnóstico, prescrição e } \\
\text { elaboração de plano de tratamento, mas admite como exceção } \\
\text { os casos em que o paciente, estando sob supervisão direta de }\end{array}$ & $\begin{array}{l}\text { Nos municípios em que há pactuação com algum } \\
\text { dos Núcleos de Telessaúde: deverá ser registrada nas } \\
\text { plataformas disponibilizadas pelos núcleos. } \\
\text { Nos municípios em que não há pactuação com } \\
\text { algum dos Núcleos de Telessaúde: } \\
\text { * Necessidade de adequação: } \\
\text { Teleaconselhamento,Teleinterconsulta, } \\
\text { Telematriciamento, Teleinterconsulta para } \\
\text { matriciamento. } \\
\text { *vale ressaltar que essas adequações são } \\
\text { administrativas, nos sistemas de informação, e não } \\
\text { geram impacto orçamentário. }\end{array}$ \\
\hline
\end{tabular}




\begin{tabular}{|l|l|l|}
\hline & $\begin{array}{l}\text { Cirurgião-Dentista, este realize troca de informações com } \\
\text { outro CD, com objetivo de prestar uma melhor assistência ao } \\
\text { paciente }{ }^{30}\end{array}$ & \\
\hline
\end{tabular}

